\title{
Evaluation of the Neurocognitive and Emotional Functions in Addicts Treated with Methadone and Buprenorphine
}

\author{
Mehdi Madanifard ${ }^{1 *}$, Mehrdad Mazaheri ${ }^{2}$ Imanolah Bigdeli ${ }^{1}$ \\ ${ }^{1}$ Department of Psychology, Faculty of Education and Psychology, Ferdowsi University of Mashhad, Mashhad, Iran \\ ${ }^{2}$ Department of Psychology, Sistan and Baluchestan University, Zahedan, Iran
}

\section{ABSTRACT}

Introduction: Cognitive and emotional factors play a crucial role in addiction treatment. The aims of this study was to evaluate cognitive emotional regulation and neurocognitive functioning in addicts treated with methadone and buprenorphine.Materials and Methods: The current study was a cross-sectional and causal-comparative study. Statistical population of this research include all men of maintenance substance treatment. The population evaluated in this study consisted of 50 patients treated with methadone and 50 patients treated with buprenorphine in Mashhad Addiction Recovery Center during 2016. The Barkley deficits in Executive Functioning Scale (BDEFS) and Garnofsky’s Emotion Regulation Scale were evaluated for all individuals. Data analysis was performed using descriptive indicators and independent t-test. Results: The results showed that the mean score in the cognitive emotion regulation scale of the buprenorphine-treated group were higher than the methadone-treated group. In contrast, the methadone-treated group received a significant higher score in BDEFS test compared to the buprenorphine group. Conclusion: Although both methadone and buprenorphine lead to a reduction in neurocognitive functioning and emotion regulation, buprenorphine seems to be a better drug to preserve cognitive function during treatment of addiction.

Key words:

1. Methadone

2. Buprenorphine

3. Emotions

*Corresponding Author: Mehdi Madanifard

E-mail:psy.madani@yahoo.com 
ارزيابى كاركردهاى عصبشناختى و هيجانى در معتادان تحت درمان با متادون و بويرنورفين

$$
\begin{aligned}
& \text { مهدى مدنى فرد'"، مهرداد مظاهرى'، ايمان الله بيكدلى' } \\
& \text { 'كروه روانشناسى، دانشكده علوم تربيتى و روانشناسى، دانشكاه فردوسى مشهد، مشهد، ايران }
\end{aligned}
$$

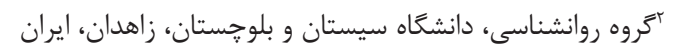

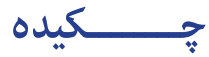

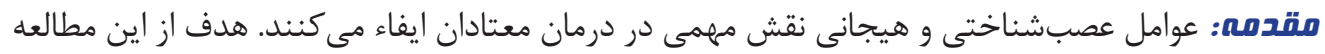

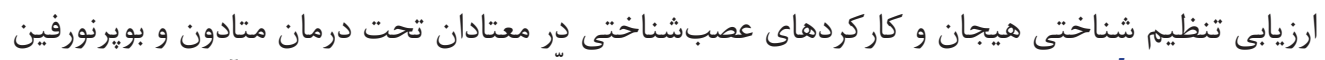

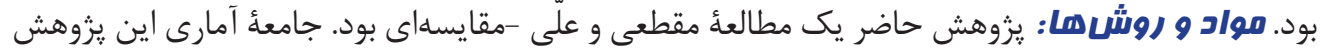

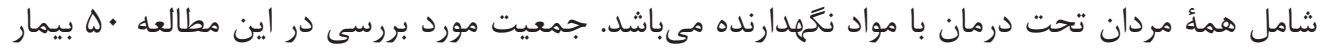

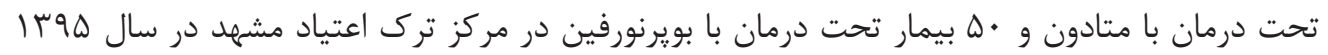

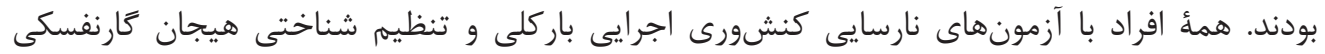

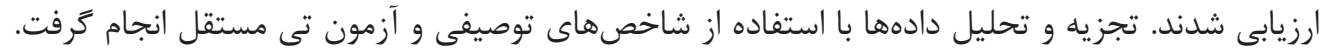

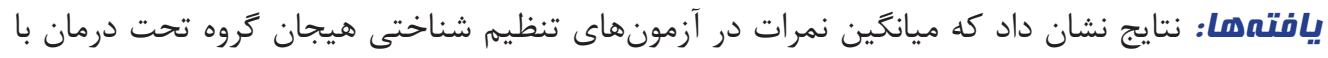

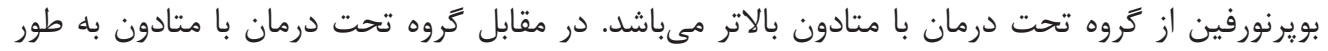

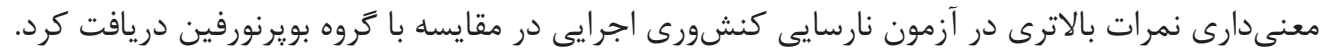

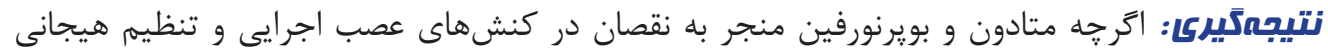

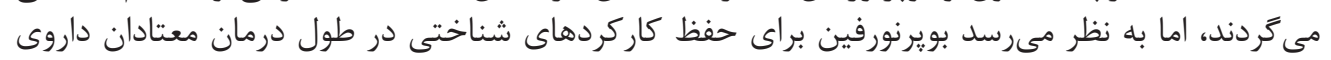
بهترى باشد.

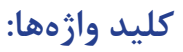
1 r. r. بوريرنورفين

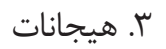


در بسـيارى از كشـورها در روشهــاى درمان و سـاختار مراقبت

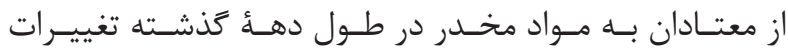

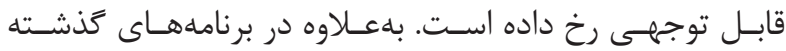

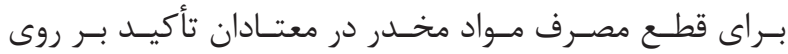

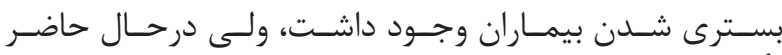

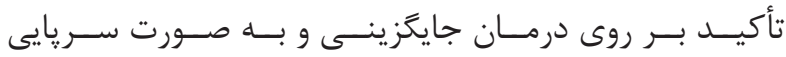

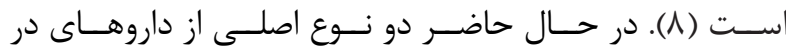

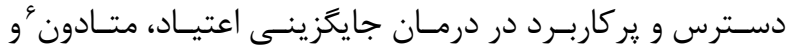

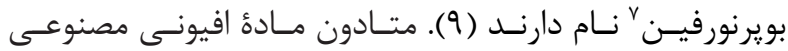

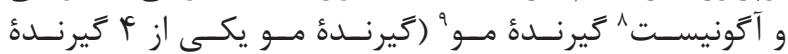

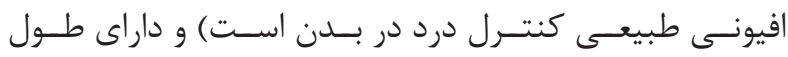

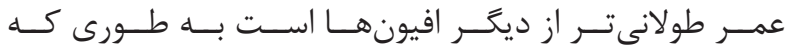

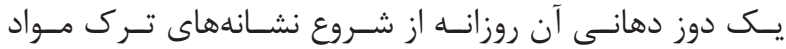

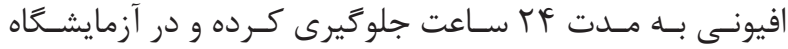

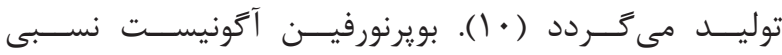

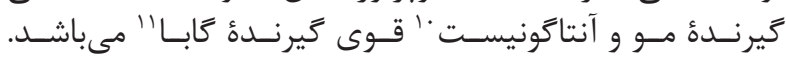

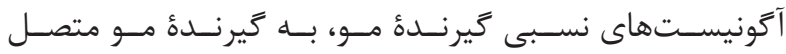

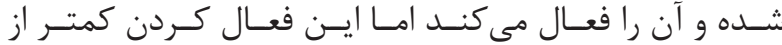

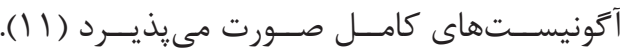

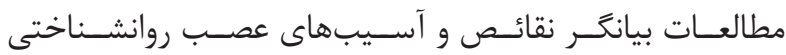

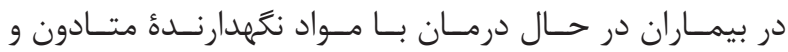

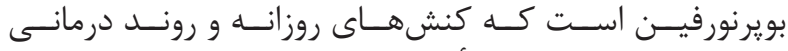

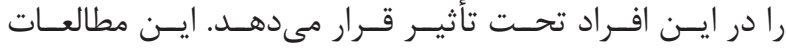

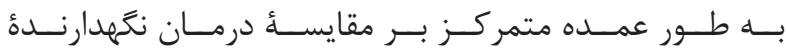

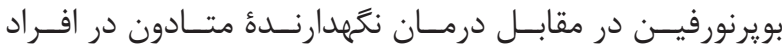

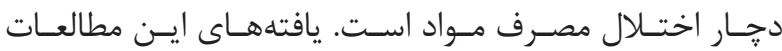

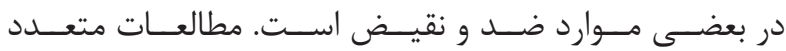

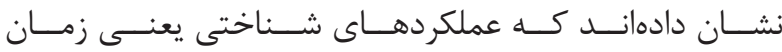

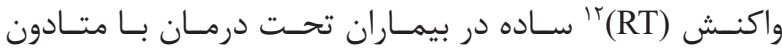

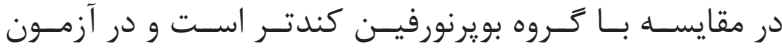

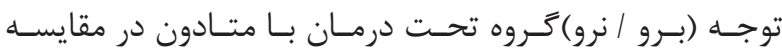

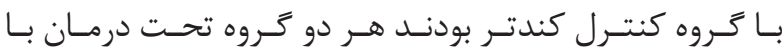

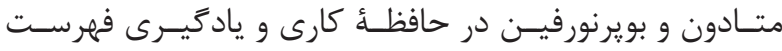

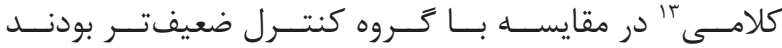

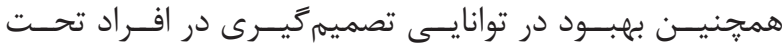

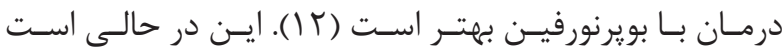

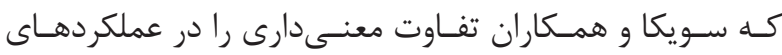

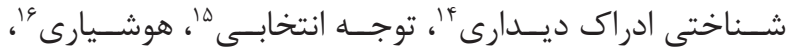

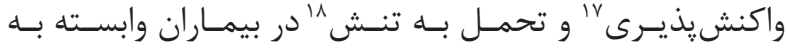

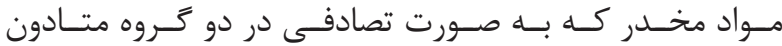

${ }^{1}$ Diagnostic of ststidtical manual of mental disorder

${ }^{2}$ Substance use disorder

${ }^{3}$ Cognitive behavior therapy

${ }^{4}$ Mindfulness based on relapse prevention

${ }^{5}$ Maintenance treatment

${ }^{6}$ Methadone

${ }^{7}$ Boupernorphine

${ }^{8}$ Agonist

${ }^{9} \mu$ receptor

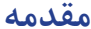

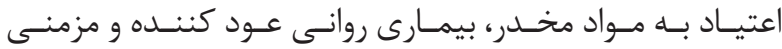

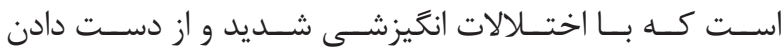

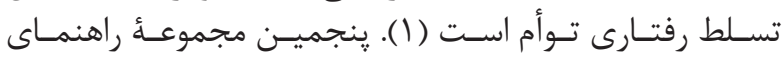

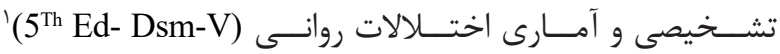

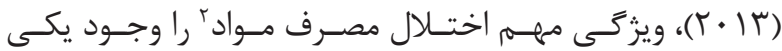

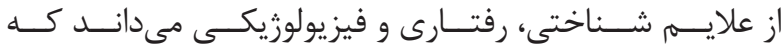

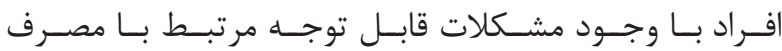

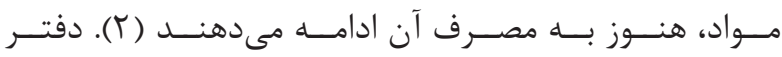

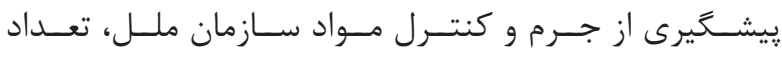

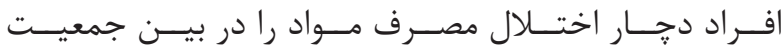

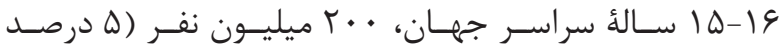

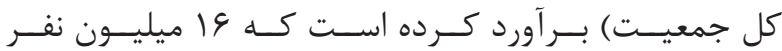

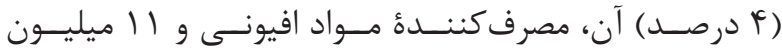

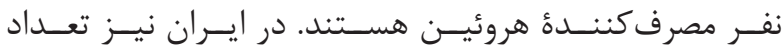

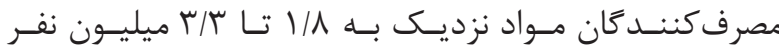

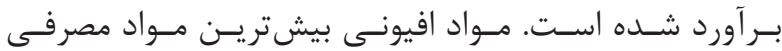

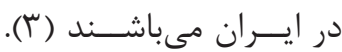

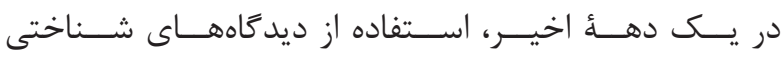

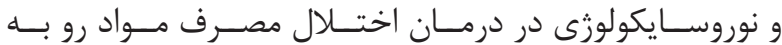

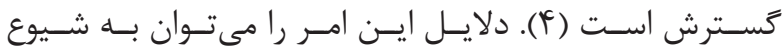

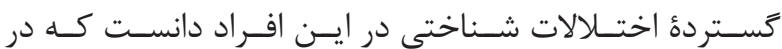

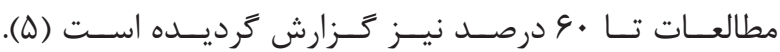

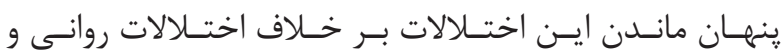

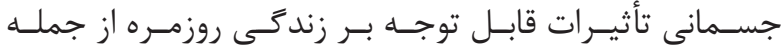

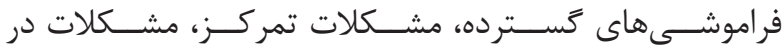

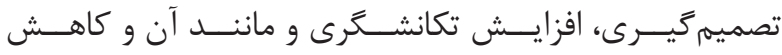

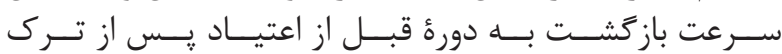

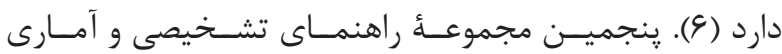

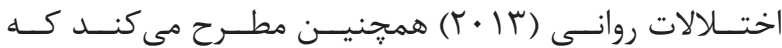

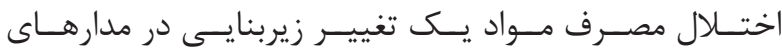

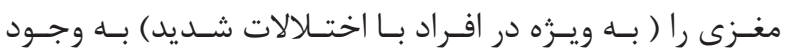

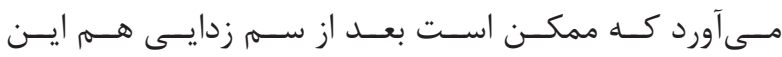

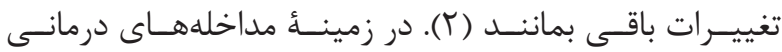

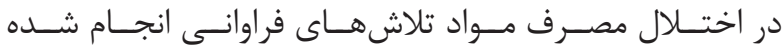

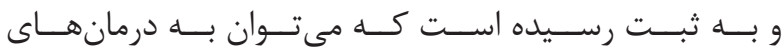

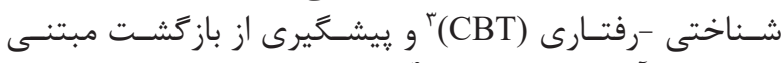

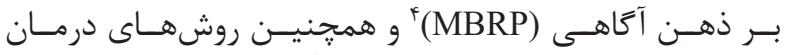

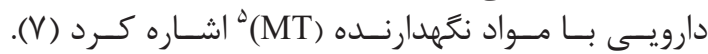
${ }^{10}$ Antagonist
${ }^{11}$ Gaba
${ }^{12}$ Reaction time
${ }^{13}$ Verbal list learning
${ }^{14}$ Visual perception
${ }^{15}$ Selective attention
${ }^{16}$ Vigilance
${ }^{17}$ Reactivity
${ }^{18}$ Stress tolerance 


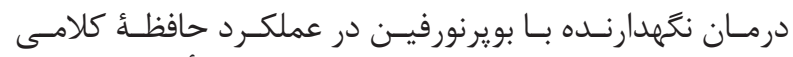

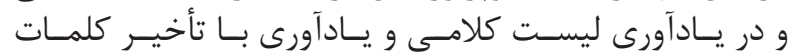

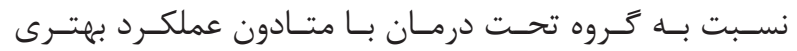

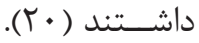

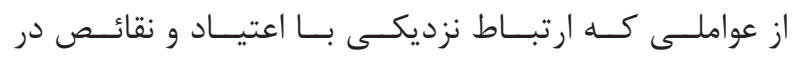

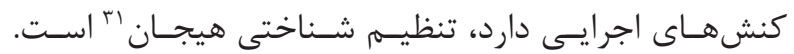

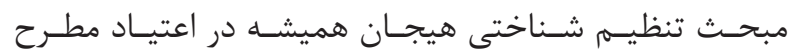

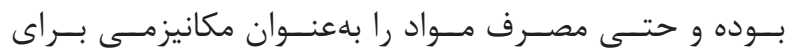

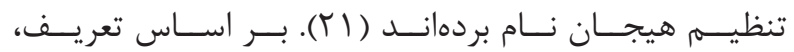

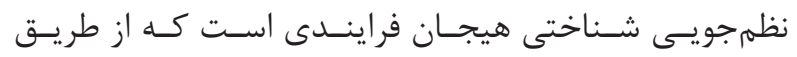

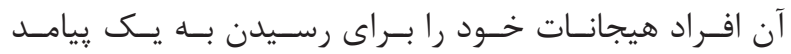

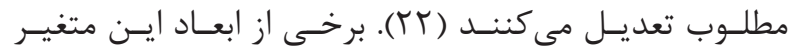

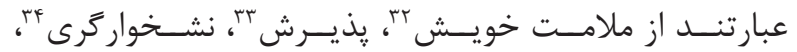

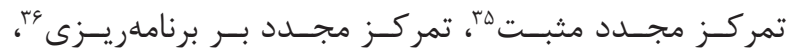

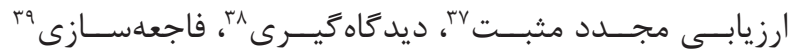

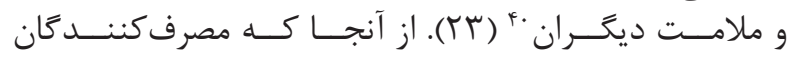

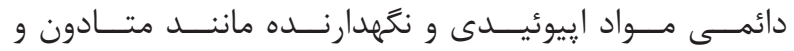

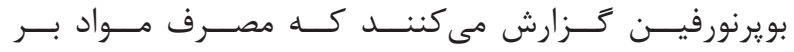

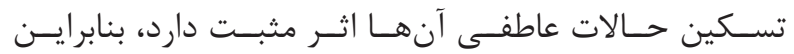

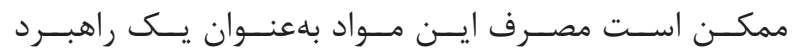

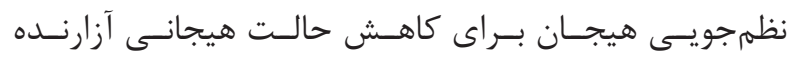

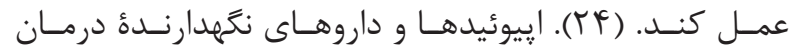

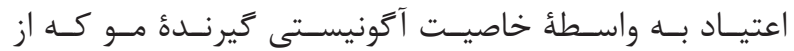

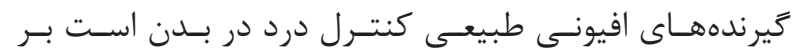

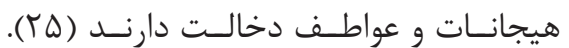

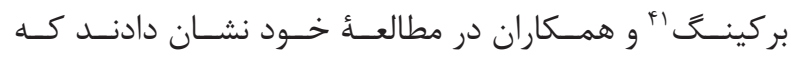

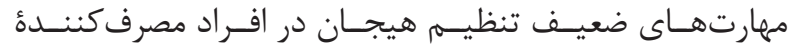

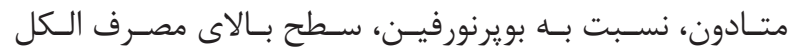

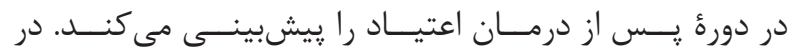

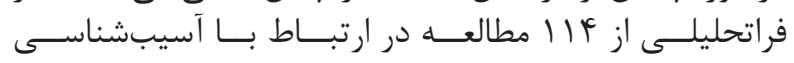

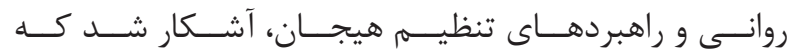

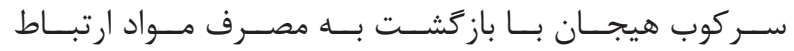

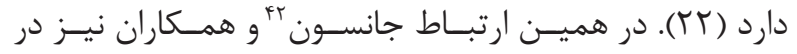

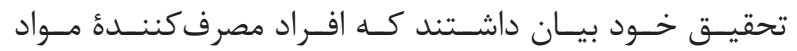

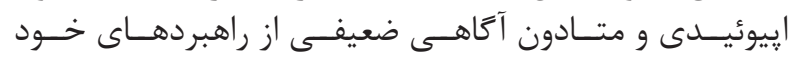

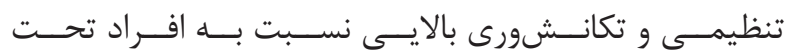

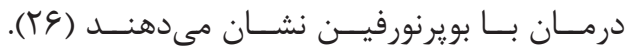

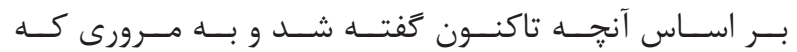

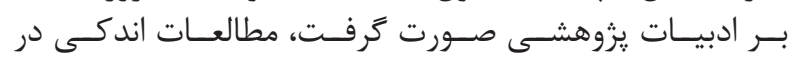

${ }^{19}$ Psychomotor performance

${ }^{20}$ Executive function

${ }^{21}$ Self-management to time

${ }^{22}$ Self-organization/problem-solving

${ }^{23}$ Self-restraint- inhibition

${ }^{24}$ Self -motivation

${ }^{25}$ Self-regulation of emotion

${ }^{26}$ Neuroanatomically

${ }^{27}$ Prefrontal cortex

${ }^{28}$ Frontal

${ }^{29}$ Darke, Sims, Mcdonald, and Wicks

${ }^{30}$ Giacomuzzi

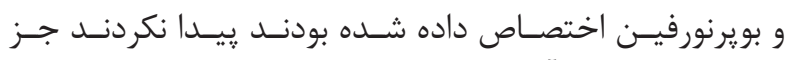

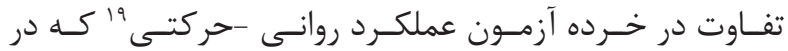

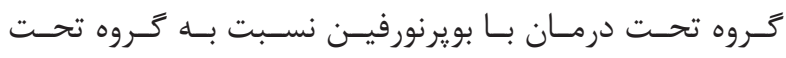

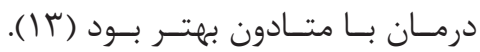

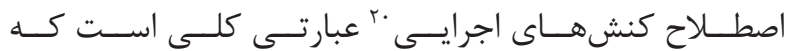

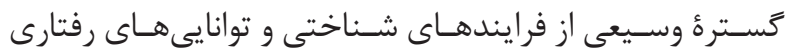

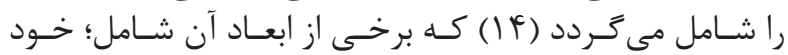

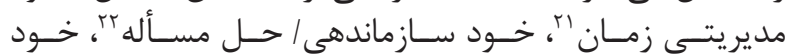

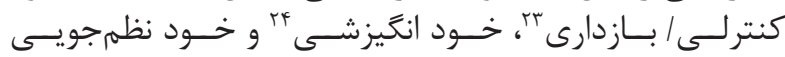

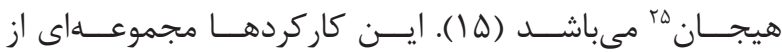

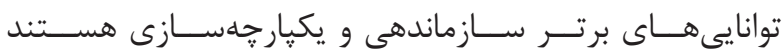

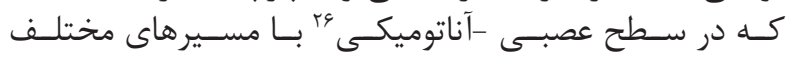

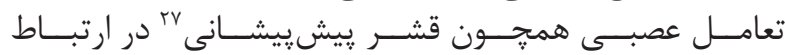

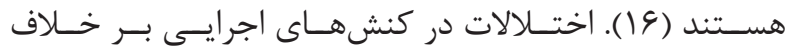

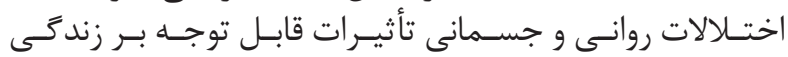

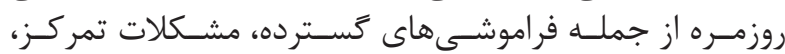

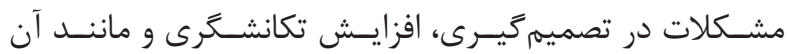

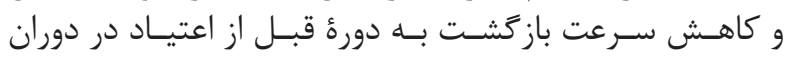

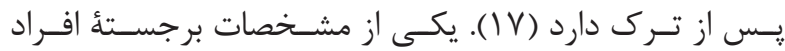

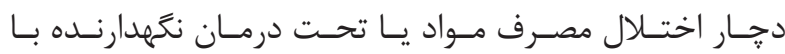

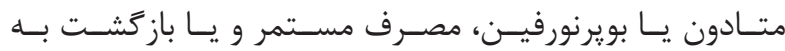

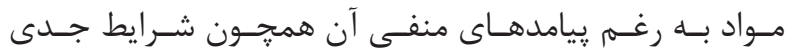

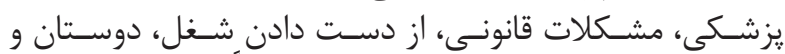

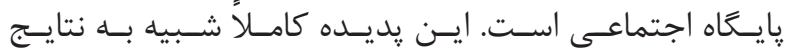

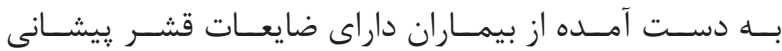

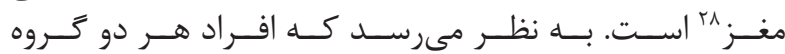

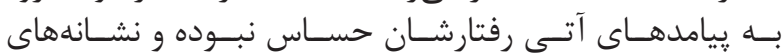

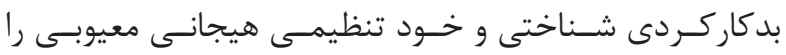

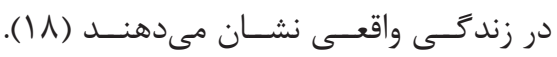

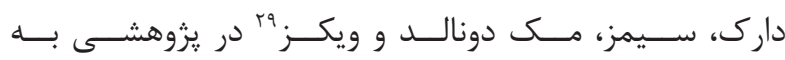

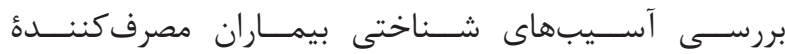

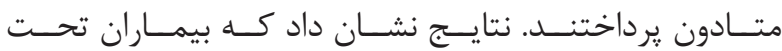

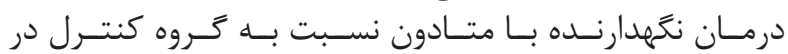

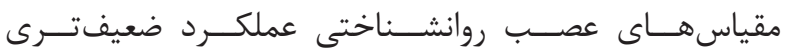

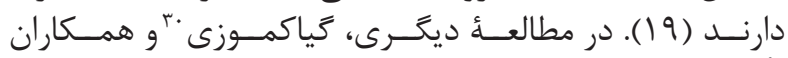

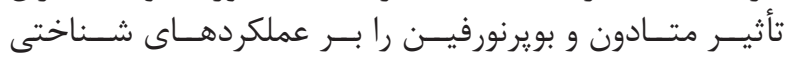

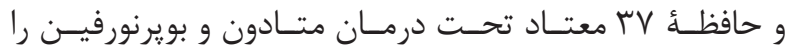

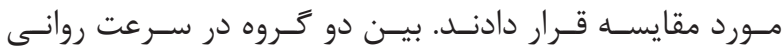

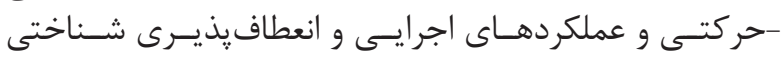

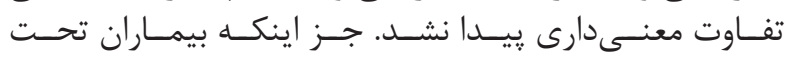

\footnotetext{
${ }^{31}$ Cognitive emotional regulation

${ }^{32}$ Self-blame

${ }^{33}$ Acceptance

${ }^{34}$ Rumination

${ }^{35}$ Positive refocusing

${ }^{36}$ Refocus on planning

${ }^{37}$ Positive reappraisal

${ }^{38}$ Putting into perspective

${ }^{39}$ Catastrophizing

${ }^{40}$ Other-blame

${ }^{41}$ Berking

${ }^{42}$ Johnson
} 


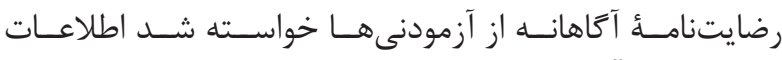

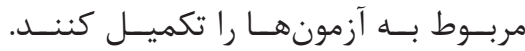

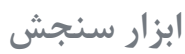

\section{بر سشنامةٔ نظمجويى شناختى هيجان گَارنفسكى}

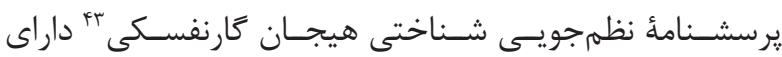

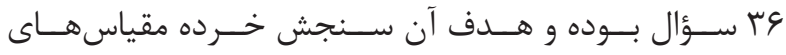

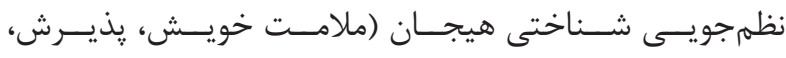

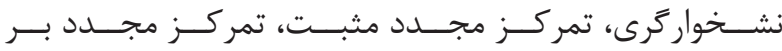

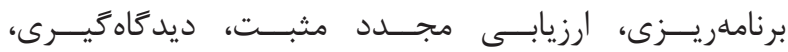

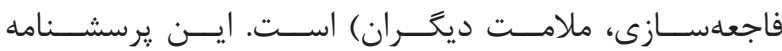

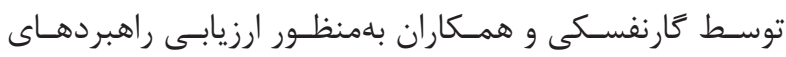

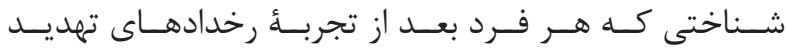

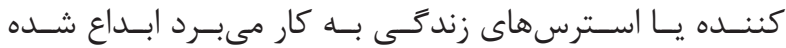

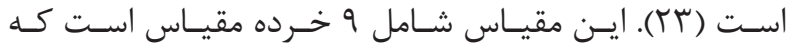

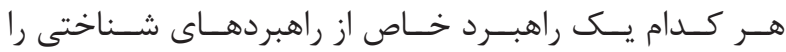

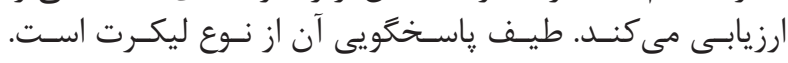

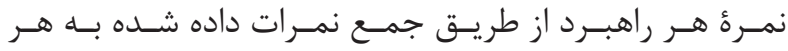

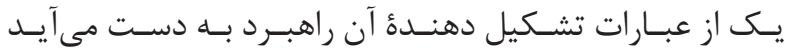

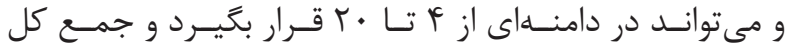

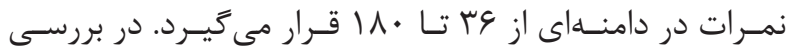

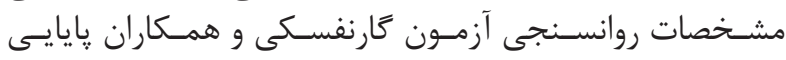

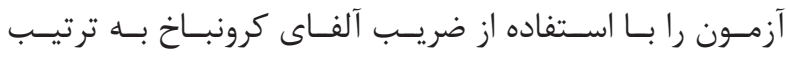

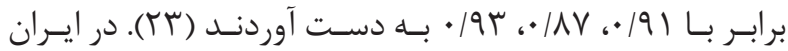

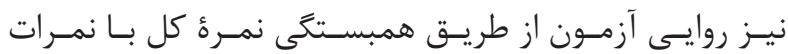

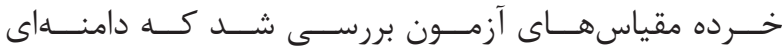

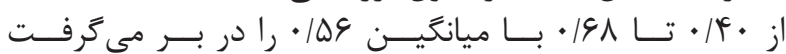

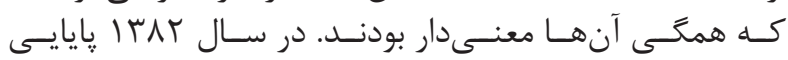

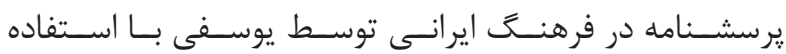

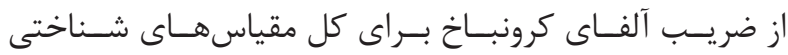

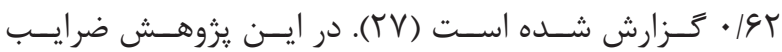

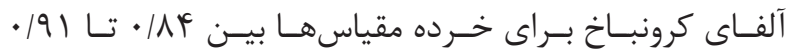

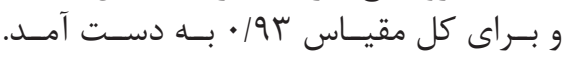

\section{يرسشنامة نارسايى كنشورى اجرايى باركلى}

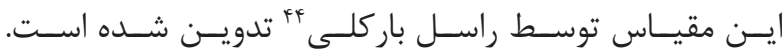

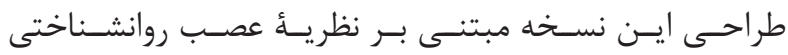

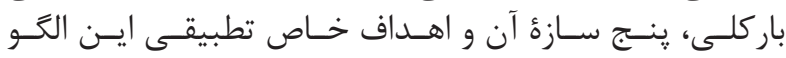

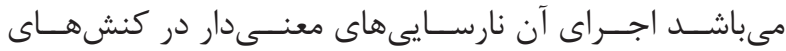

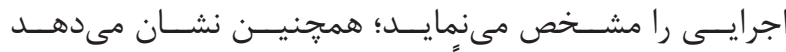

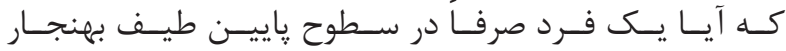

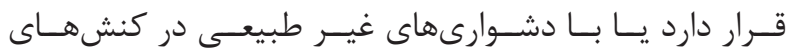

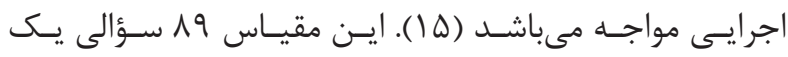

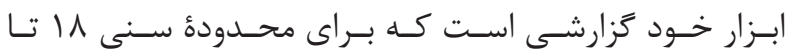

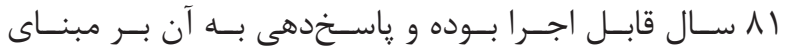

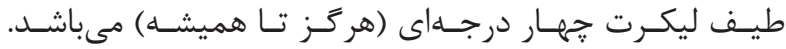

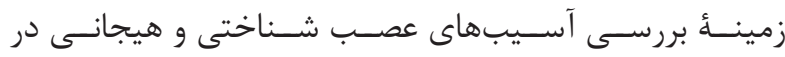

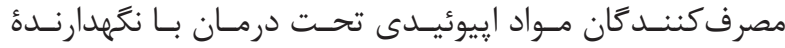

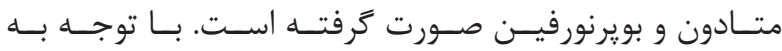

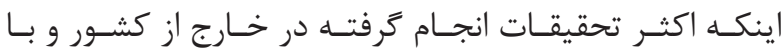

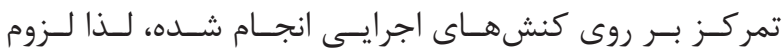

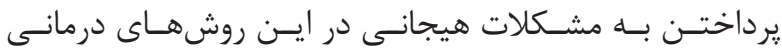

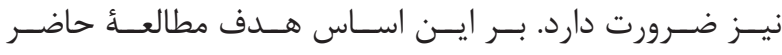

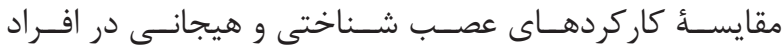

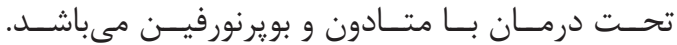

$$
\text { مواد و روشها }
$$

روش يثوهش، جامعلة آمارى و نمونه

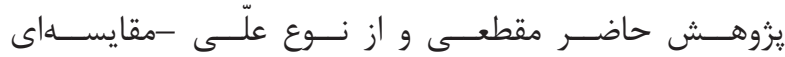

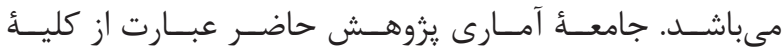

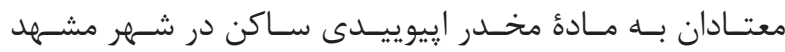

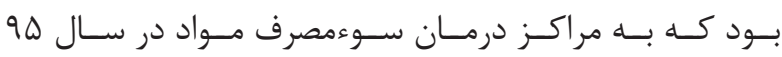

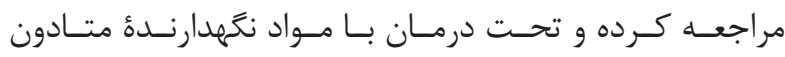

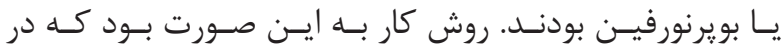

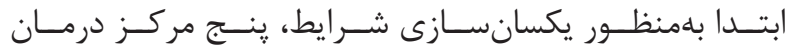

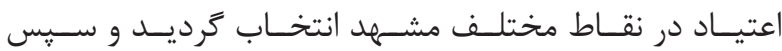

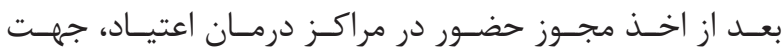

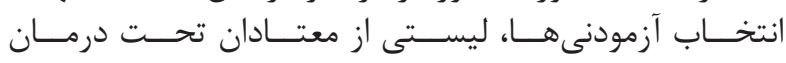

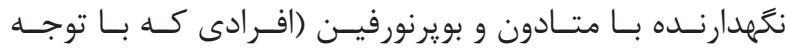

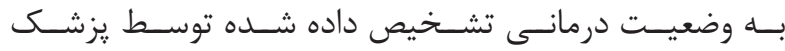

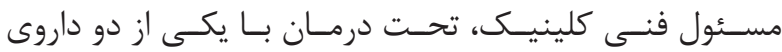

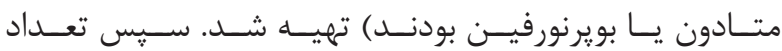

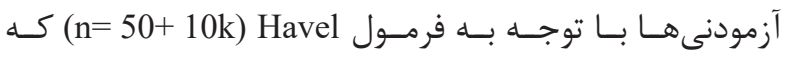

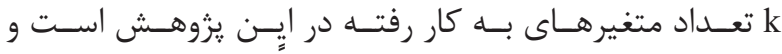

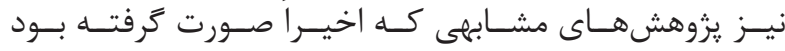

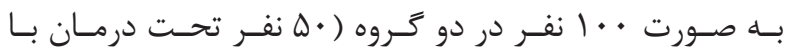

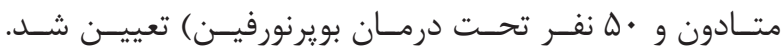

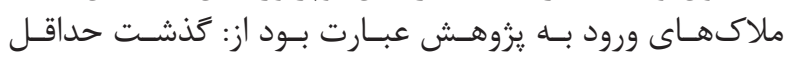

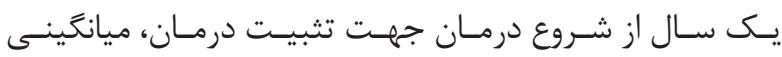

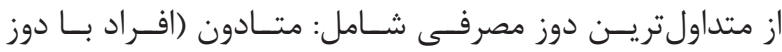

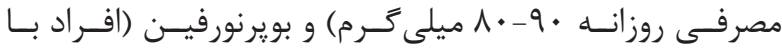

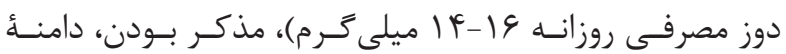

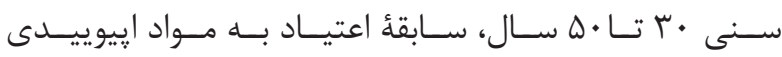

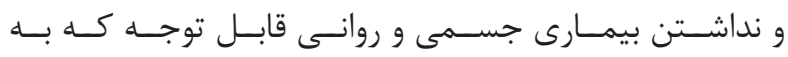

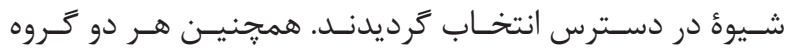

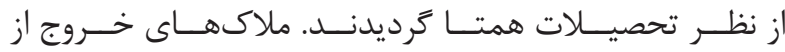

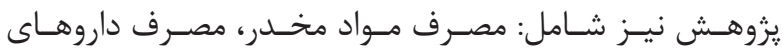

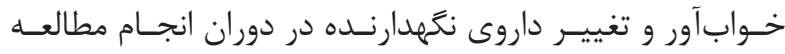

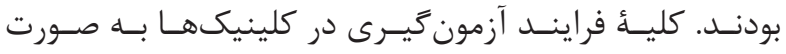

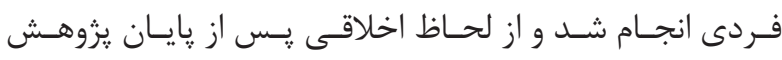

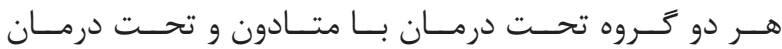

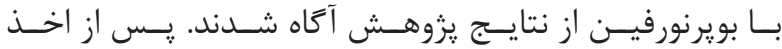

${ }^{43}$ Garnefski

${ }^{44}$ Barkley 


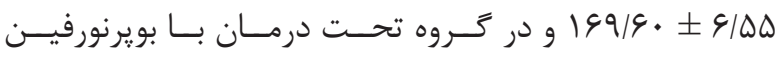

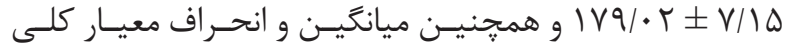

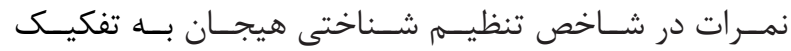

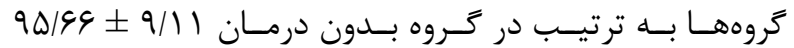

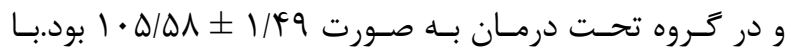

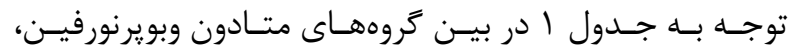

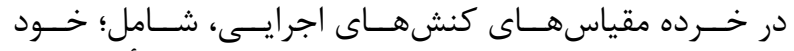

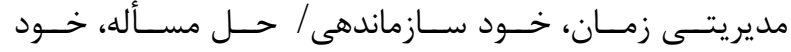

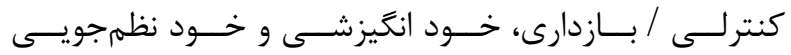

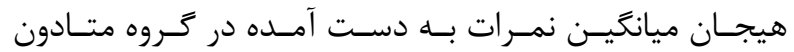

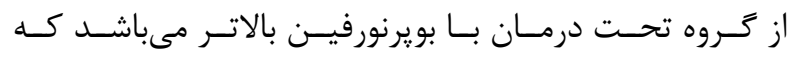

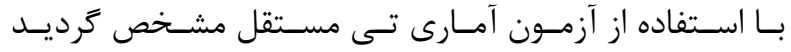

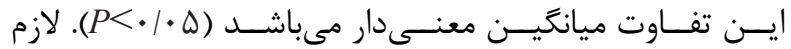

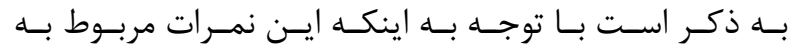

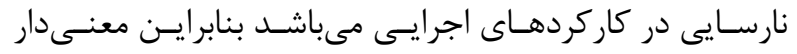

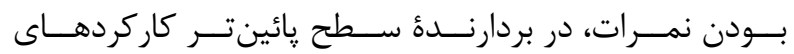

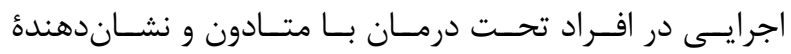

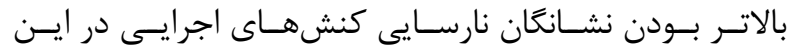

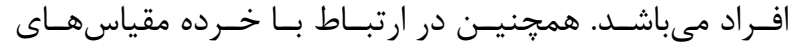

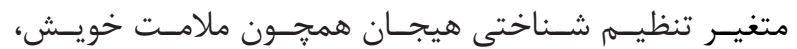

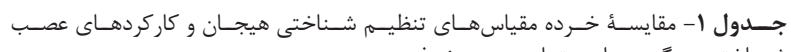

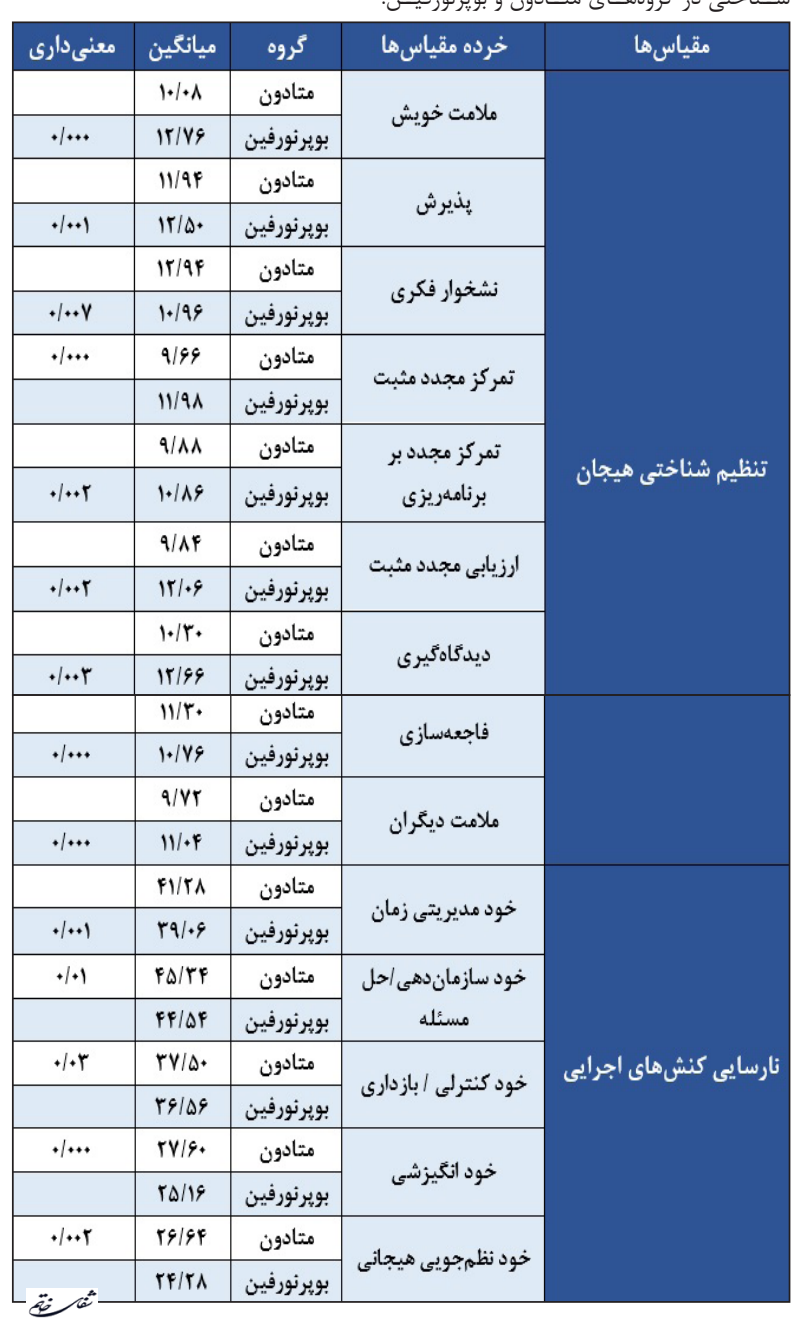

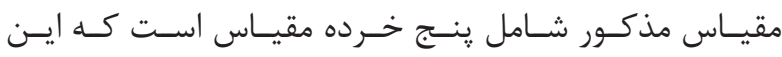

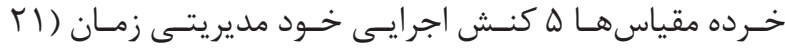

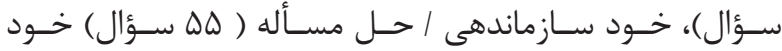

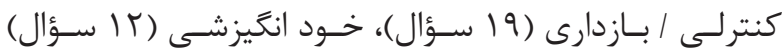

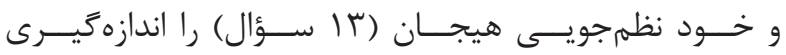

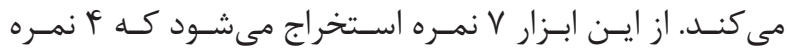

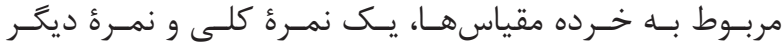

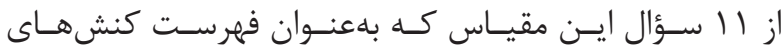

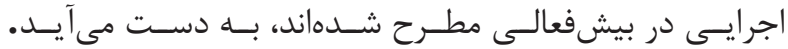

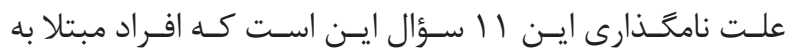

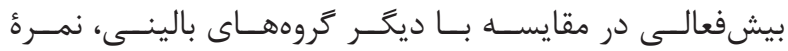

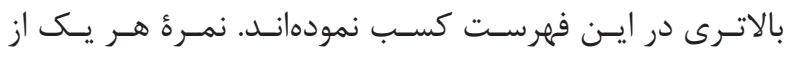

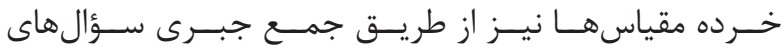

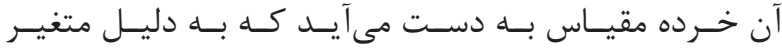

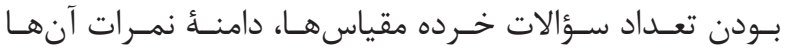

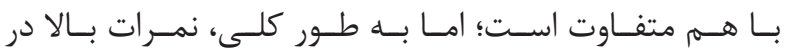

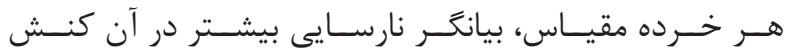

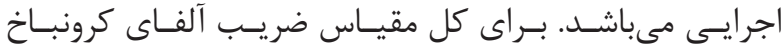

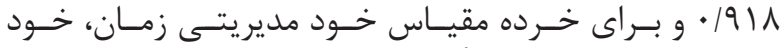

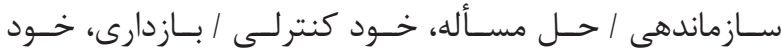

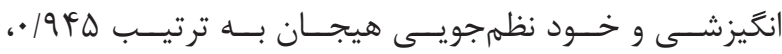

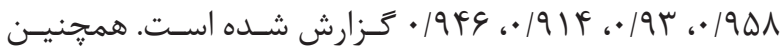

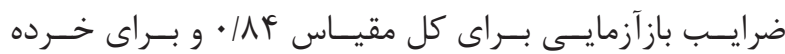

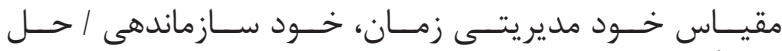

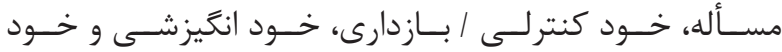

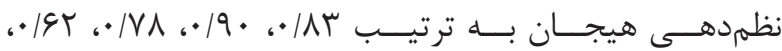

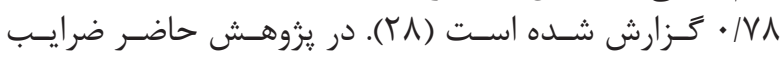

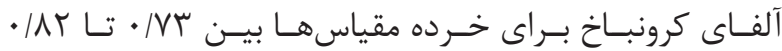

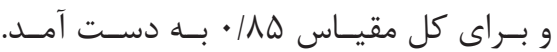

تجزيه و تحليل دادهها

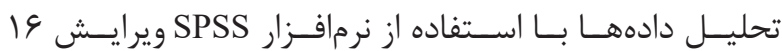

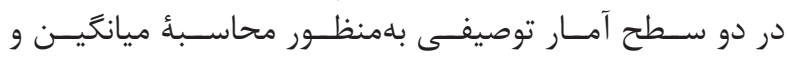

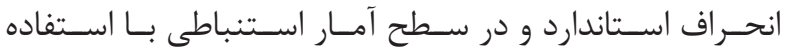

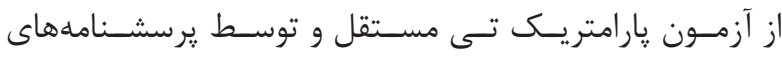

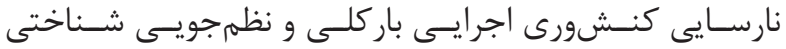

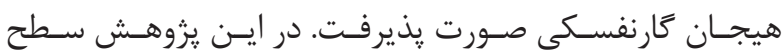

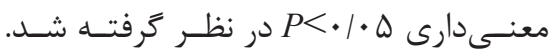

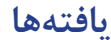

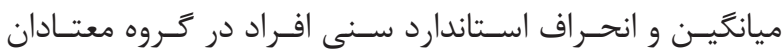

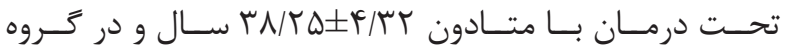

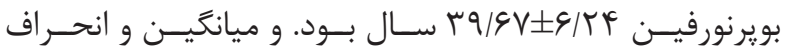

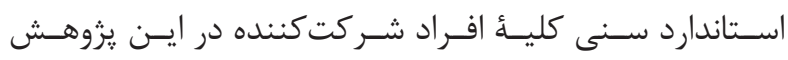

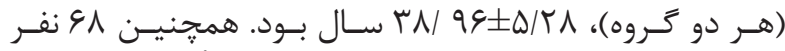

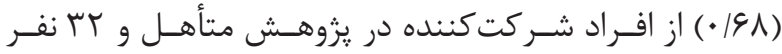

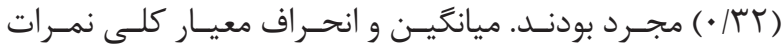

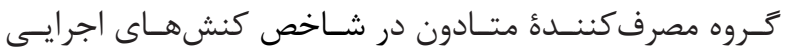




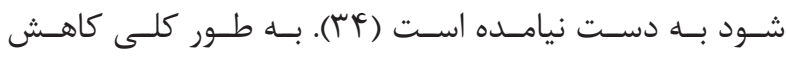

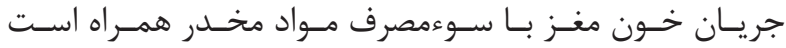

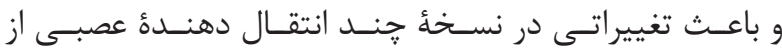

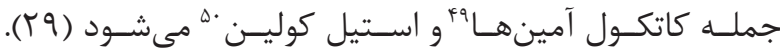

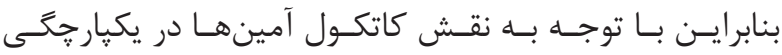

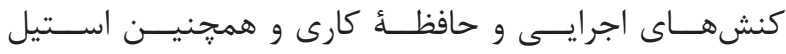

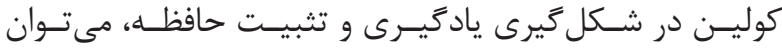

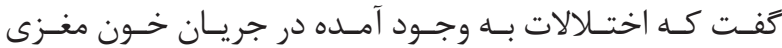

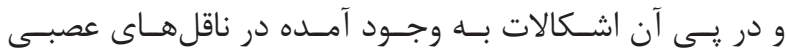

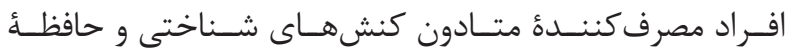

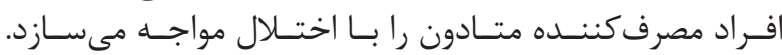

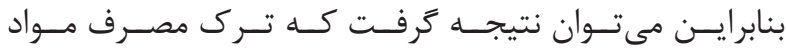

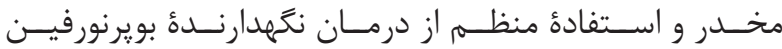

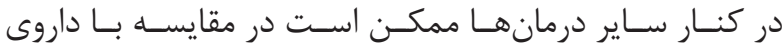

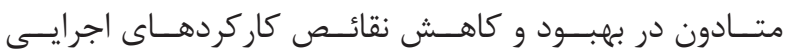

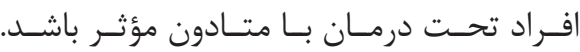

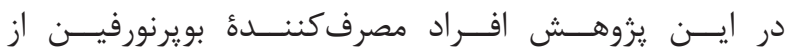

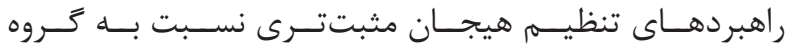

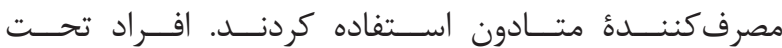

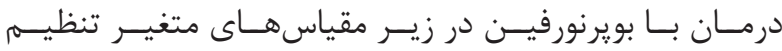

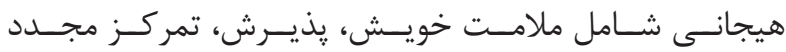

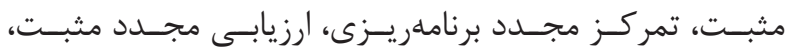

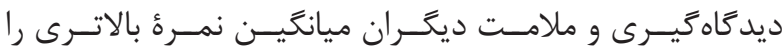

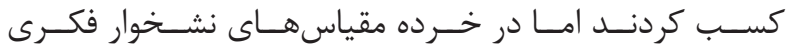

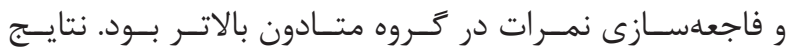

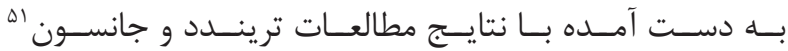
بr)

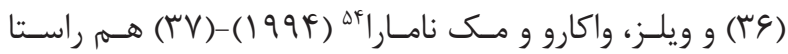

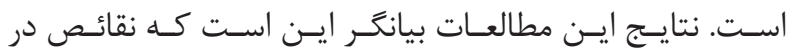

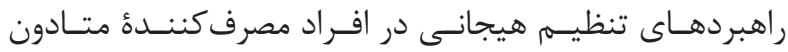

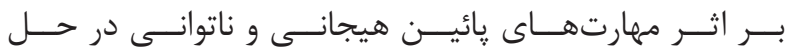

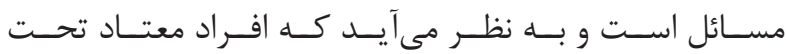

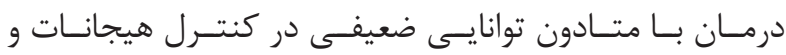

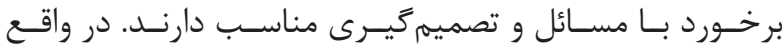

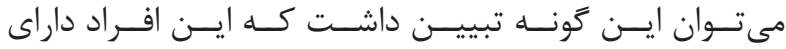

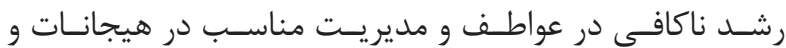

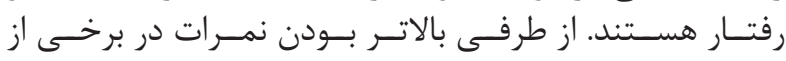

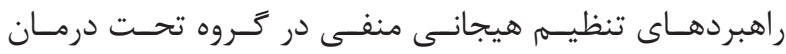

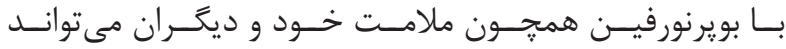

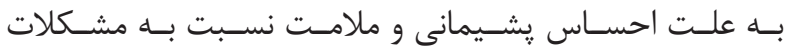

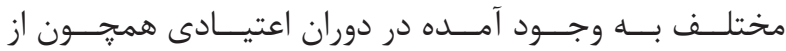

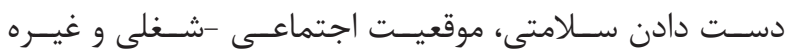

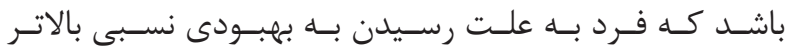

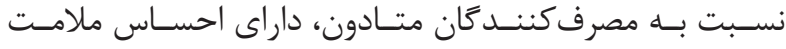

${ }^{45}$ Lundqvist

${ }^{46}$ Von Geusau

${ }^{47}$ Soyka

${ }^{48}$ Ventrolateral prefrontal cortex

${ }^{49}$ Catecholamine

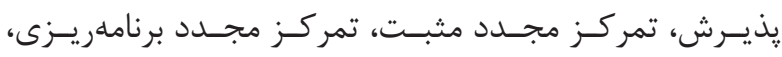

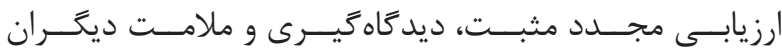

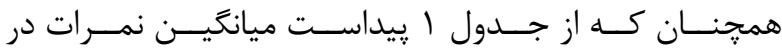

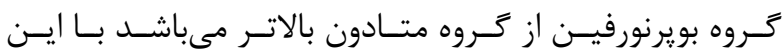

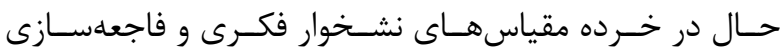

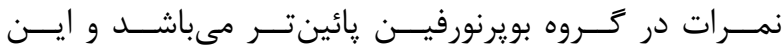

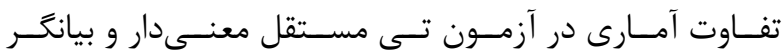

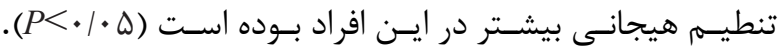

\section{بحث و نتيجه}

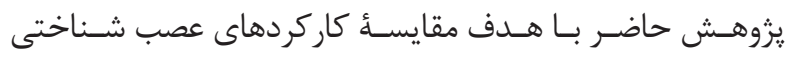

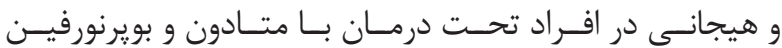

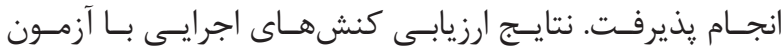

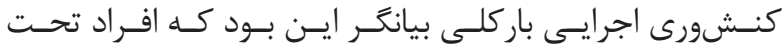

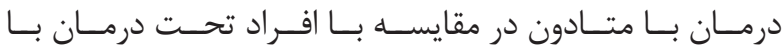

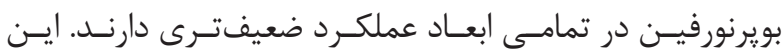

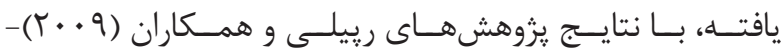

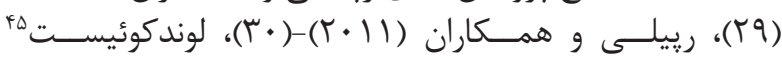
(Y)

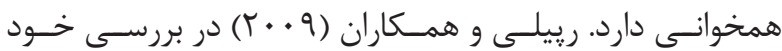

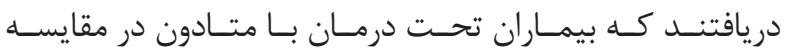

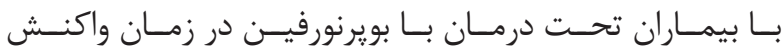

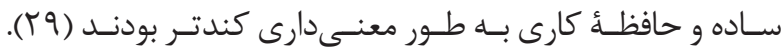

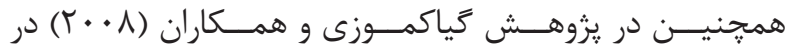

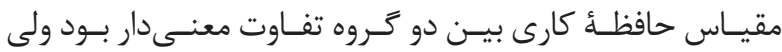

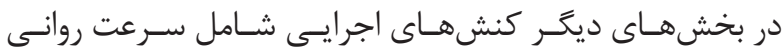

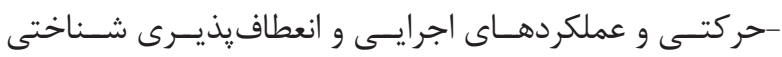

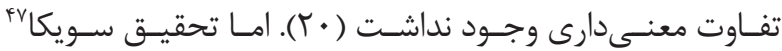

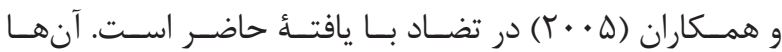

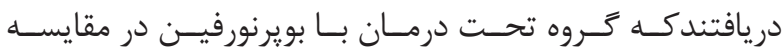

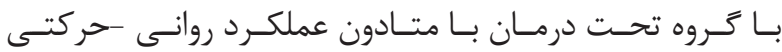

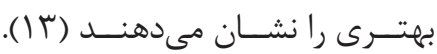

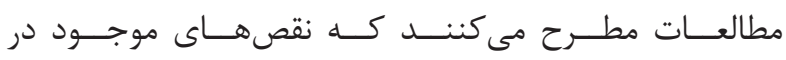

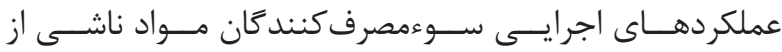

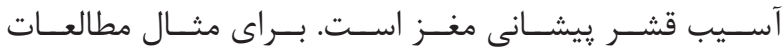

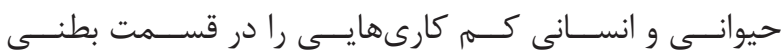

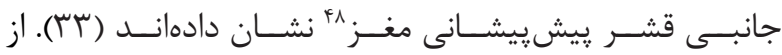

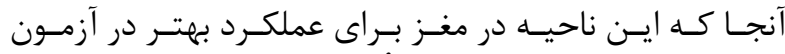

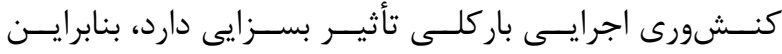

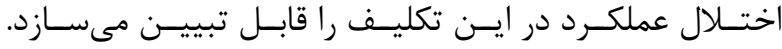

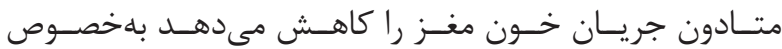

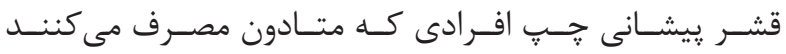

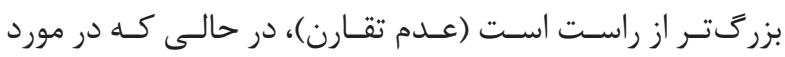

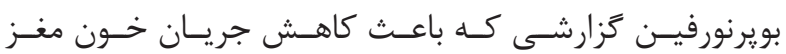
${ }^{50}$ Acetylcholine
${ }^{51}$ Trindad and Johnson
${ }^{52}$ Unger
${ }^{53}$ Chou
${ }^{54}$ Wills, Vaccaro and Mcnamara 
كوشـش در زمينـــُ مهــار و كنتــرل هيجانـات و احساسـات

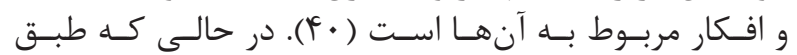

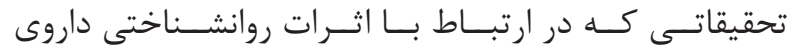

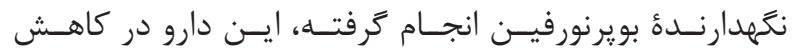

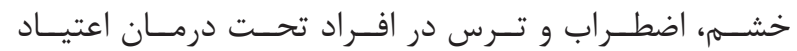

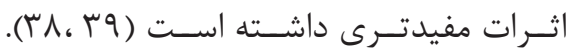

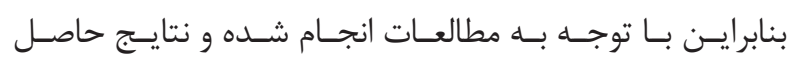

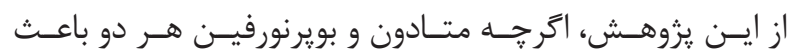

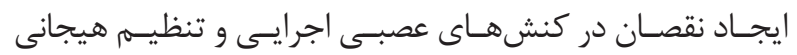

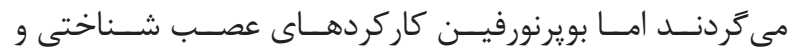

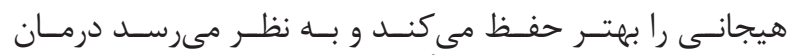

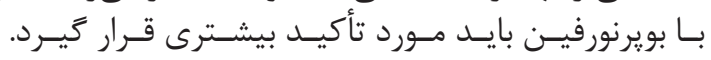

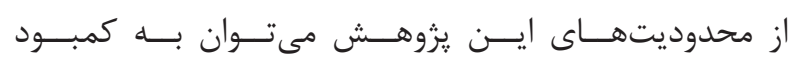

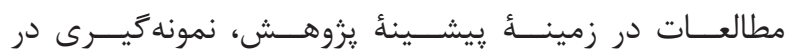

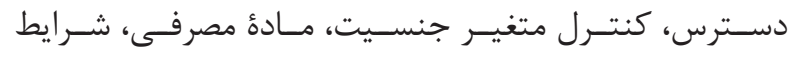

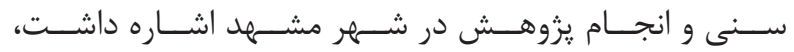

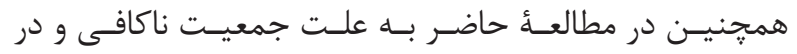

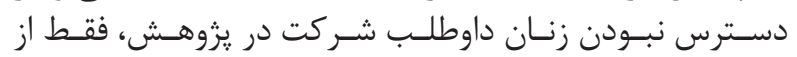

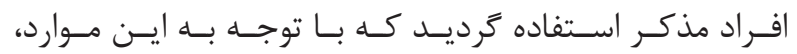

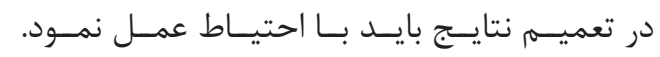

1. Dallas TX, David W, Julie K. Staley behavioral neuroscience of drug addiction. Biosocieties. 2010; 39: 22-6.

2. American Psychiatric Association. Diagnostic and statistical manual of mental disorders. $5^{\text {Th }}$ ed. Washington, DC: APA. 2013.

3. Mokri A. Brief overview of the status of drug abuse in Iran. Archives of Iranian Medicine. 2002; 5: 184-90.

4. Paulus MP, Hozack NE, Zauscher BE, Frank L, Brown GG, Braff DL. Behavioral and functional neuroimaging evidence for prefrontal dysfunction in methamphetaminedependent subjects. Neuropsychopharmacology. 2002; 26(1): 53-6.

5. Lyvers G, Leggio L, Abenavoli L, Gasbarrini G. Neurobiochemical and clinical aspects of craving in alcohol addiction: a review. Addict Behav. 2005; 30(6): 1209-24.

6. Czuchry M, Dansereau DF. Cognitive skills training: impact on drug abuse counseling and readiness for treatment. Am J Drug Alcohol Abuse. 2003; 29(1): 1-18.

7. Witkiewitz K, Marlatt A, Walker D. Ming-fullnessbased relapse prevention for alcohol and substance use

\footnotetext{
${ }^{55}$ Ipser

${ }^{56}$ Electrooculography
}

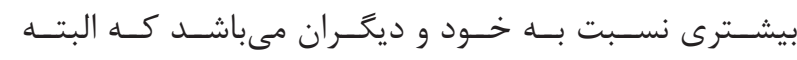

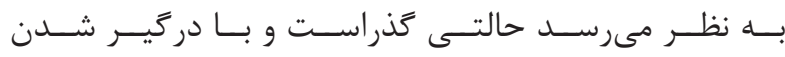

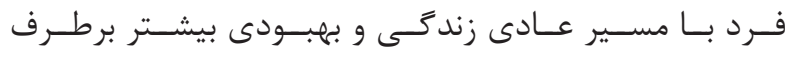

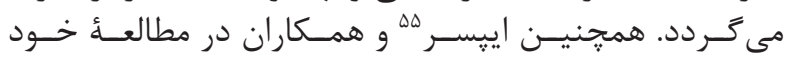

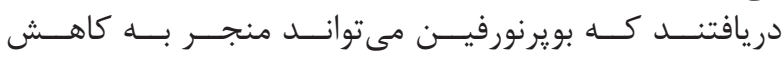

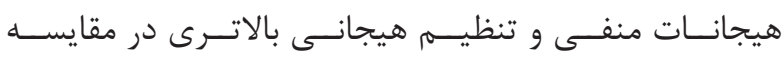

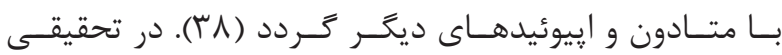

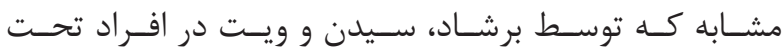

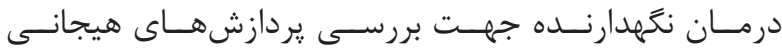

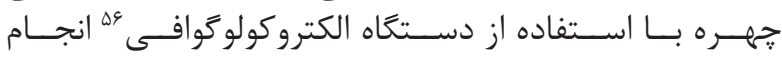

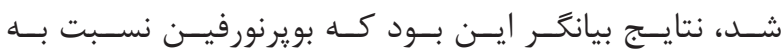

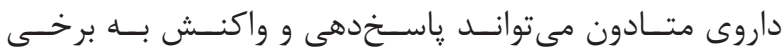

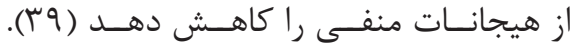

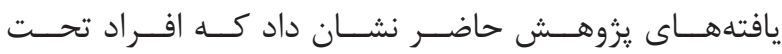

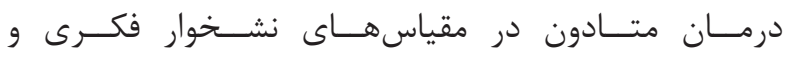

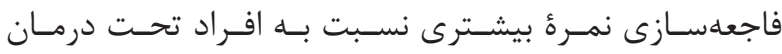

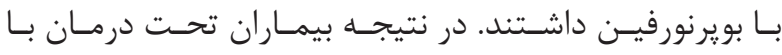

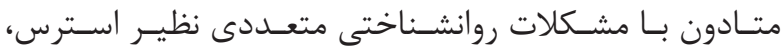

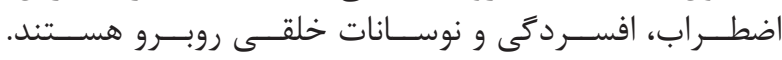

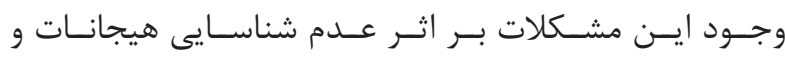

منابع

disorders. J Cogn Psychother. 2005; 19: 221-2.

8. McLellan AG, Arndt IO, Metzger DS, Woody GE, O'Brien CP. The effects of psychosocial services in subs tance abuse treatment. JAMA. 1993; 269(15): 1953-9.

9. Layson-Wolf C, Goode JV, Small RE. Clinical use of methadone. J Pain Palliat Care Pharmacother. 2002; 16(1): 29-59.

10. Connock M, Juarez-Garcia A, Jowett S, Frew E, Liu $\mathrm{Z}$, Taylor RJ, et al. Methadone and buprenorphine for the management of opioid dependence: a systematic review and economic evaluation. Health Technol Assess. 2007; 11(9):1-171.

11. Huizinga M, Dolan C, Van Der Molen M. Agerelated change in executive function: developmental trends and a latent variable analysis. Neuropsychol. 2006; 44(11): 2017-36.

12. Soyka M, Horak M, Dittert S, Kagerer S. Less driving impairment on buprenorphine than methadone in drug-dependent patients? J Neuropsychiatry Clin Neurosci. 2001; 13(4): 527-8.

13. Soyka M, Hock B, Kagerer S, Lehnert R, Limmer $\mathrm{C}$, Kuefner H. Less impairment on one portion of a 
driving-relevant psychomotor battery in buprenorphinemaintained than in methadone-maintained patients. results of a randomized clinical trial. $\mathrm{J}$ Clin Psychopharmacol. 2005; 25(5): 490-3.

14. Gerra G, Borella F, Zaimovic A, Moi G, Bussandri $\mathrm{M}$, Bubici C, et al. Buprenorphine versus methadone for opioid dependence: predictor variables for treatment outcome. Drug Alcohol Depend. 2004; 75(1): 37-45.

15. Barkley RA. Deficits in executive functioning scale (BDEFS). New York: Guilford Press; 2011.

16. Lyvers G, Leggio L, Abenavoli L, Gasbarrini G. Neurobiochemical and clinical aspects of craving in alcohol addiction: a review. Addictive Behaviors. 2005; 30(6): 1209-24.

17. Lezak MD. Neuropsychological assessment. $3^{\text {rd }}$ ed. New York: Oxford University Press. 1995.

18. Bechara A, Dolan S, Denburg N, Hindes A, Anderson SW. Nathan PE. Decision-making deficits, linked to a dysfunctional ventromedial prefrontal cortex, revealed in alcohol a stimulant abuser. Neuropsychologia. 2001; 39(4): 376-89.

19. Darke S, Sims J, Mcdonald S, Wicks W. Cognitive impairment among methadone maintenance patients. Addiction. 2000; 95(5): 687-95.

20. Giacomuzzi SM, Thill C, Riemer Y, Garber K, Ertl M. Buprenorphine-and methadone maintenance treatment: influence on aspects of cognitive and memory performance. Open Addict J. 2008; 1: 5-6.

21. Taube-Schiff M, Van Exan J, Tanaka R, Wnuk S, Hawa R, Sockalingam S. "Attachment style and emotional eating in bariatric surgery candidates: the mediating role of difficulties in emotion regulation". Eat Behav. 2015; 18: 36-40.

22. Aldao A, Nolen-Hoeksema S, Schweizer S. Emotionregulation strategies across psychopathology: a metaanalytic review. Clin Psychol Rev. 2010; 30(2): 217-37.

23. Garnefski N, Teerds J, Kraaij V, Legerstee J, Van den Kommer T. Cognitive emotion regulation strategies and depressive symptoms: differences between males and females. Pers Individ Dif. 2004; 36(2): 267-76.

24. Barlow DH, Allen LB, Choate ML. Toward a unified treatment for emotional disorders. Behav Ther. 2004; 35(2): 205-30.

25. Savvas S, Somogyi A, White J. The effect of methadone on emotional reactivity. Addiction.
2012;107(2): $\quad 388-92$.

26. Rolley E. Pharm D. Mary C. Eric S. Shahron W. Maxiline L. et al. A comparison of 1 evomethadyl acetate, buprenorphine, and methadone for opioid dependence. $\mathrm{N}$ Engl J Med. 2000; 324(2): 1290-4.

27. Yusefi F. The relationship of cognitive emotion regulation strategies with depression and anexiety in the middle students. Except Child. 2007; 4: 871-92.

28. Mashhadi A, Mirdoraghi F, Hosainzadeh-Maleki Z, Hasani J, Hamzeloo M. Factor structore, reliability and validity of persian version of barkeley deficits in executive functioning scale(BDEFS)- adult version. J Clin Psychol. 2015; 7(1): 51-62.

29. Rapeli P, Carola Fabritius C, Kalska H, Alho H. Memory function in opioid dependent patients treated with methadone or buprenorphine along with benzodiazepine: longitudinal change in comparison to healthy individuals. Subst Abuse Treat Prev Policy. 2009; 4: 6. doi:10.1186/1747-597X-4-6.

30. Rapeli P, Carola Fabritius C, Kalska H, Alho H. Cognitive functioning in opioid dependentpatients treated with buprenorphine, methadone, and other psychoactive medications: stability and correlates. BMC Clin Pharmacol. 2011; 11: 13. doi.org/10.1186/14726904-11-13.

31. Lundqvist T. Cognitive consequences of connobese use in comparison with abuse of stimulants and heroin with regard to attention, memory and executive functions. Pharmacol Biochem Behav. 2005; 81(2): 319-30.

32. Von Geusau NA, Stalenhoef P, Huizinga M, Snel J, Ridderinkhof KR. Impaired executive function in male MDMA ("ecstasy") users. Psychopharmacology (Berl). 2004; 175(3): 331-441.

33. Hampshire A, Owen AM. Fractionating attentional control using eventrelated fmri. Cereb Cortex. 2006; 16(12): $1679-89$.

34. Rapeli P, Fabritius C, Alho H, Salaspuro M, Wahlbeck K, Kalska H. Methadone vs buprenorphine/ naloxone during early opioid substitution treatment: a naturalistic comparison of cognitive performancerelative to healthy controls. BMC Clin Pharmacol. 2007; 7: 5. doi.org/10.1186/1472-6904-7-5.

35. Trindad DR, Unger Jb, Chou CO, Johnson A. The protective association of emotional intellegence with psychosocial smoking risking factors factor adolescent. personality and individual differences. Pers Individ Dif. 2004; 36(4): 945-54. 


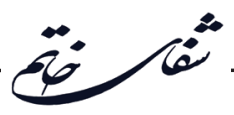

36. Trindad DR, Johnson CA. The association between emotion intelligence and early adolescent tobaco and alcohol use. Pers Individ Dif. 2002; 3(21): 95-105.

37. Wills TA, Vaccaro D, Mcnamara G. Novehty seeking, risk taking and related constructs as predictors of adolescent substance use: an application of cloninger s theory. J Subst Abuse. 1994; 6(1): 1-20

38. Ipser JC1, Terburg D, Syal S, Phillips N, Solms M, Panksepp J, et al. Reduced fear-recognition sensitivity following acute buprenorphine administration in healthy volunteers. Psychoneuroendocrinology. 2013; 38(1): $166-70$.

39. Bershad AK, Seiden JA, de Wit H. Effects of buprenorphine on responses to social stimuli in healthy adults. Psychoneuroendocrinology. 2016; 63: 43-9.

40. Narimani M, Abasi M, Abolghasemi A, Ahadi B. Comparison of the effectiveness of acceptance / commitment with emotion regulation training on adjus tment of students with dyscalculia. Middle Eastern Journal of Disability Studies. 2013; 4(2): 174-6. 